\title{
Stereotactic body radiation therapy for non-small cell lung cancer patients with prior history of thoracic surgery and/or radiation therapy: the influence of smoking, size, and central location on risk of complications
}

\author{
Jason W. Chan ${ }^{1}$ - Julian Johnson ${ }^{1}$ • Ann A. Lazar ${ }^{2}$ - Steve E. Braunstein ${ }^{1}$. \\ Martina Descovich $^{1}$ - Alexander R. Gottschalk ${ }^{1}$ - Sue S. Yom ${ }^{1}$
}

Received: 12 September 2017 / Accepted: 3 October 2017 /Published online: 10 October 2017

(C) The Author(s) 2017, corrected publication 2019

\begin{abstract}
Purpose The purpose of this retrospective cohort study was to evaluate the effect of tumor location and other major factors on the risk of complications from stereotactic body radiation therapy (SBRT) in non-small cell lung cancer (NSCLC) patients with a history of thoracic surgery and/or radiation therapy.

Methods Medical records of patients with recurrent or second primary NSCLC treated with SBRT between 2005 and 2015 were reviewed. All patients had prior thoracic surgery and/or radiation therapy and were treated with image-guided, robotic SBRT. Time to local failure and toxicity was evaluated by using cumulative incidence and competing risk regression analysis. Overall survival was estimated using the Kaplan-Meier method. Results Fifty-six patients with 74 tumors treated with SBRT were included for analysis. Of the 74 tumors, 19 (26\%) were centrally located within $2 \mathrm{~cm}$ of the proximal bronchial tree. At a median follow-up of 27 months (range $=5-129$ ), the 2-year cumulative incidence of LF was $13 \%$ for peripheral tumors and $16 \%$ for central tumors $(p=0.95)$. Neither prior thoracic surgery nor radiation therapy was associated with increased LF or toxicity. Among the 56 patients, eight (14\%), three (5\%), and one (2\%) experienced grade 2,3 , and 4 complications following SBRT. Patients with $\mathrm{a} \geq 50$ pack-year smoking history were more likely to develop $\geq$ grade 2 toxicity (HR 5.3; 95\% CI 1.4
\end{abstract}

This work has not been previously presented or published in any form.

Sue S. Yom

sue.yom@ucsf.edu

1 Department of Radiation Oncology, Helen Diller Comprehensive Cancer Care Center, University of California, San Francisco, 1600 Divisadero St., Suite H-1031, San Francisco, CA 94143-1708, USA

2 Department of Preventive and Restorative Dental Sciences, University of California, San Francisco, San Francisco, CA, USA
19.9, $p=0.01)$. Central tumor location was associated with higher risk of toxicity (HR 3.4; 95\% CI 1.1-10.5, $p=0.04$ ) as was planning tumor volume $>30 \mathrm{~mL}$ (HR $4.3 ; 95 \%$ CI $1.1-$ $16.3, p=0.047)$. In a multivariable model, central location remained significant after adjusting for smoking history and planning tumor volume.

Conclusions After prior thoracic surgery and/or radiation, SBRT retreatment for NSCLC provided durable local control and was well tolerated in most patients. Patients with a history of previously operated and/or radiated lung cancer with new central tumors, PTV volume $>30 \mathrm{~mL}$, or smoking history $\geq 50$ pack-years were at higher risk of complications from SBRT.

Keywords Stereotactic body radiation therapy $\cdot$ Non-small cell lung cancer $\cdot$ Retreatment $\cdot$ Central $\cdot$ Peripheral $\cdot$ Smoking

\section{Introduction}

The ideal treatment approach in previously treated lung cancer patients who experience a locoregional failure or a new primary non-small cell lung cancer (NSCLC) is not well defined. This is a common situation, as the risk for developing new primary lung cancers is approximately $2 \%$ per year in NSCLC patients and is correlated with pack-year smoking history [1]. Operable patients may be treated with salvage surgical resection, but among patients who initially receive radiation therapy for lung cancer treatment, they were often not surgical candidates to begin with. Conventionally, fractionated external beam radiation (EBRT) given for salvage intent is associated with poor tumor control [2], but more recent single-institutional series show that stereotactic body radiation therapy (SBRT) provides superior results [3]. In patients who recur after previous thoracic radiation therapy, reirradiation with SBRT appears to be mostly 
safe, but isolated incidents of toxicity have been observed with retreatment of centrally located tumors [4].

The safety of treating centrally located tumors with SBRT is becoming established, especially among medically inoperable patients with early-stage NSCLC where SBRT is accepted as a first-line standard of care [5]. In one of the first SBRT studies in lung cancer, a regimen of 54 Gy in three fractions yielded excellent local control exceeding $90 \%$, but an 11-fold increase in grades 3-5 adverse events was observed for tumors located within $2 \mathrm{~cm}$ of the central airway [6]. As a result of this and other corroborating studies, central lung tumors were excluded from multi-institutional clinical trials, such as RTOG 0236 and RTOG 0618, and at present, many institutions favor SBRT of central lung tumors with extended fractionation [7], including ours, where a five-fraction course has been used for most centrally located tumors. Early presentations of efficacy and safety data from the phase I/II dose-finding study NRGRTOG 0813 support this approach for central tumors, with reported 2-year local control rates of 87.7-89.4\%, 2-year overall survival rates of $70.2-72.7 \%$, and raw grade 3 toxicity frequencies of 5-9\% at doses of 57.5-60 Gy over five fractions [8]. However, these data were obtained in newly diagnosed, protocol-appropriate patients, whereas safe regimens for those who have recurred or developed a second primary remain largely uncharacterized.

We noticed that the majority of complications in our thoracic SBRT practice have occurred in patients with a prior history of lung-directed cancer treatment. In this study, we report the results of central and peripheral SBRT for the retreatment of patients with recurrent or second primary NSCLC who have previously had thoracic surgery and/or radiation therapy. Our primary objective was to evaluate the effect of tumor location and other major factors on the safety and efficacy of SBRT.

\section{Methods}

\section{Patient and tumor characteristics}

This retrospective cohort study was based on a review of all patients treated with SBRT for recurrent or new primary NSCLC at a single institution from 2005 to 2015. Waiver of consent for medical chart review was approved by the Institutional Review Board (study number 10-05156).

Smoking history was measured in pack-years, which is the product of the packs of cigarettes smoked per day and the number of years the person has smoked. According to the NRG-RTOG definition, centrally located lung tumors were defined as being within $2 \mathrm{~cm}$ of the carina, bilateral main bronchi, bilateral upper lobe bronchi, intermedius bronchus, right middle lobe bronchus, lingular bronchus, or bilateral lower lobe bronchi.
Initial patient evaluations included $\mathrm{CT}$ of the chest with contrast, FDG-PET/CT scan, pulmonary function testing, and biopsy unless medically contraindicated or refused by the patient. Follow-up imaging consisted of serial CT of the chest with contrast every 3 months. Interval growth on CT prompted FDG-PET/CT imaging.

Follow-up time was calculated from the date of SBRT completion. Local failures (LF) were defined as post-SBRT recurrences within $2 \mathrm{~cm}$ of the original tumor extent. These represented new or progressing lesions within or at the margin of the PTV. LF was determined jointly by the treating radiation oncologist and diagnostic radiologist, but histologic confirmation was not required. Toxicity grading was according to the National Cancer Institute Common Terminology Criteria for Adverse Events, version 4. Toxicity grading from follow-up and hospitalization records were independently performed by two raters to ensure reliability. Grade 1 toxicities were not consistently recorded and were therefore not included in our analyses.

\section{Radiation treatment}

All patients were treated with real-time image-guided SBRT planned and delivered on a robotic SBRT system (CyberKnife Robotic Radiosurgery System, Accuray Inc., Sunnyvale, CA). Within the constraints of this system, only six MV photons were used in treatment planning. Following the publication of the TG-101 report [9], our institution adopted these dose constraints. Before the TG-101 report, constraints were more stringent. Prior to 2010, the ray tracing algorithm was used for dose calculations. After 2010, planners used Monte Carlo dose calculations. The differences in individual beam dose calculations for ray tracing versus Monte Carlo calculations were not studied in this cohort. TG-135 was followed for quality assurance.

\section{Statistical analyses}

Patients with central versus peripheral tumors were compared using chi-square for categorical covariates and Wilcoxon rank-sum tests for covariates measured on a continuous scale. Time to local failure and $\geq$ grade 2 toxicity was evaluated by using cumulative incidence analysis (Gray's test) and competing risk regression analysis to estimate subdistribution hazard ratios (HRs) and 95\% confidence intervals (CIs) treating death without local failure as a competing event [10]. Overall survival was estimated using the Kaplan-Meier method and Cox proportional hazards model $[11,12]$. Covariates with $p$ value $\leq 0.1$ on univariable analysis were entered into a multivariable competing risk regression model. Due to a low number of local failure events, multivariable models could not be generated for this endpoint. Two-sided $p$ values $<0.05$ were considered significant. Statistical analyses were performed using $\mathrm{R}$, version 3.3.1. 


\section{Results}

\section{Patient, tumor, and treatment characteristics}

Of 65 patients fitting the initial study eligibility criteria, nine did not have follow-up imaging after SBRT and were excluded from analysis. None of these nine patients experienced severe adverse reactions to SBRT. The remaining 56 fully evaluable patients had a total of 74 tumors that were retreated

Table 1 Characteristics of 56 patients with history of non-small cell lung cancer retreated with stereotactic body radiation therapy

\begin{tabular}{|c|c|c|}
\hline & Central & Peripheral \\
\hline Number of patients & 14 & 42 \\
\hline \multicolumn{3}{|l|}{ Sex } \\
\hline Male (\%) & $7(50)$ & $19(45)$ \\
\hline Female $(\%)$ & $7(50)$ & $23(55)$ \\
\hline \multicolumn{3}{|l|}{ Age (years) } \\
\hline Median & 66 & 73 \\
\hline Range & $54-81$ & $52-89$ \\
\hline \multicolumn{3}{|l|}{ KPS } \\
\hline Median & 80 & 80 \\
\hline Range & $80-90$ & $60-100$ \\
\hline \multicolumn{3}{|l|}{ COPD } \\
\hline Yes $(\%)$ & $11(79)$ & $27(64)$ \\
\hline No $(\%)$ & $3(21)$ & $15(36)$ \\
\hline \multicolumn{3}{|l|}{ Smoking history (pack-years) } \\
\hline Median & 35 & 40 \\
\hline Range & $20-120$ & $0-100$ \\
\hline$\geq 50$ pack-year smoking $(\%)$ & $5(36)$ & $23(55)$ \\
\hline$\geq 20$ pack-year smoking $(\%)$ & $11(79)$ & $30(71)$ \\
\hline Active smoker $(\%)$ & $2(14)$ & $10(24)$ \\
\hline \multicolumn{3}{|c|}{ Interval since initial treatment (years) } \\
\hline Median & 4.2 & 2.9 \\
\hline Range & $0.9-9.9$ & $0.2-28.2$ \\
\hline Prior thoracic radiation $(\%)$ & $4(29)$ & $23(55)$ \\
\hline SBRT (\%) & $1(7)$ & $11(26)$ \\
\hline EBRT $(\%)$ & $3(21)$ & $12(29)$ \\
\hline Prior surgery (\%) & $13(93)$ & $27(64)$ \\
\hline Wedge or segmentectomy & $0(0)$ & $4(10)$ \\
\hline Lobectomy & $12(86)$ & $22(52)$ \\
\hline Pneumonectomy & $1(7)$ & $1(2)$ \\
\hline \multicolumn{3}{|l|}{ Number of lesions } \\
\hline Single $(\%)$ & $9(64)$ & $34(81)$ \\
\hline Multiple (\%) & $5(36)$ & $8(19)$ \\
\hline Median & 1 & 1 \\
\hline Range & $1-3$ & $1-3$ \\
\hline
\end{tabular}

KPS Karnofsky performance status, COPD chronic obstructive pulmonary disease, $S B R T$ stereotactic body radiation therapy, EBRT external beam radiation therapy
Table 2 Characteristics of 74 tumors in patients with history of nonsmall cell lung cancer receiving retreatment stereotactic body radiation therapy

\begin{tabular}{lll}
\hline & Central & Peripheral \\
\hline $\begin{array}{l}\text { Number of tumors } \\
\text { Histology }\end{array}$ & 19 & 55 \\
$\quad$ Squamous cell carcinoma (\%) & $1(5)$ & $10(18)$ \\
$\quad$ Adenocarcinoma (\%) & $18(95)$ & $45(82)$ \\
Ipsilateral thorax as site of initial primary & & \\
Yes (\%) & $8(42)$ & $31(56)$ \\
No (\%) & $11(58)$ & $24(44)$ \\
Location & & \\
Right lower lobe (\%) & $1(5)$ & $15(27)$ \\
Right upper lobe (\%) & $3(16)$ & $12(22)$ \\
Right hilum (\%) & $0(0)$ & $1(2)$ \\
Right middle lobe (\%) & $1(5)$ & $3(5)$ \\
Left lower lobe (\%) & $3(16)$ & $12(22)$ \\
Left upper lobe (\%) & $2(11)$ & $12(22)$ \\
Left hilum (\%) & $0(0)$ & $0(0)$ \\
Lingula (\%) & $1(5)$ & $0(0)$ \\
Mediastinum $(\%)$ & $6(32)$ & $0(0)$ \\
\hline
\end{tabular}

with SBRT. Patient, tumor, and treatment characteristics of the cohort are outlined in Tables 1,2, 3 .

In the 56 evaluable patients, 14 were treated for central tumors and 42 were treated for peripheral lung tumors (Table 1). Among those with central versus peripheral tumors, patient characteristics were similar, with a median age of 66 versus 73 years, respectively, and median KPS of 80 for both groups. The majority of patients had a

Table 3 Characteristics of stereotactic body radiation therapy retreatment in patients with history of non-small cell lung cancer, among 74 treated lesions

\begin{tabular}{lll}
\hline & Central & Peripheral \\
\hline PTV (mL) & & \\
Median & 28 & 19 \\
Range & $4-104$ & $1-272$ \\
Number of fractions \\
$\quad$ Median & 5 & 4 \\
Range & $3-5$ & $1-10$ \\
Isodose line $(\%)$ & \\
Median & 61 & 62 \\
Range & $38-75$ & $50-91$ \\
SBRT duration $($ days $)$ & \\
Median & 7 & 5 \\
Range & $4-10$ & $1-14$ \\
BED & & \\
Median & 100 & 100 \\
Range & $36-151$ & $60-180$ \\
\hline
\end{tabular}

$P T V$ planning tumor volume, $S B R T$ stereotactic body radiation therapy 
substantial smoking history, with more than $70 \%$ in both groups reporting $\geq 20$ pack-years of smoking and a similar proportion in both groups carrying a diagnosis of COPD. Five of the patients with central tumors had multiple lesions treated, all of which were centrally located. Three patients with central tumors were treated to two sites and two were treated to three sites. Four patients with peripheral tumors were treated to two sites and four were treated to three sites. There was no difference in the proportion of multiple-site treatments $(p=0.62)$ in central versus peripheral patients. More patients with peripheral tumors had a history of prior radiation (55 versus 29\%) whereas more patients with central tumors had a history of thoracic surgery (93 versus 64\%). In both groups, the most common prior surgery was lobectomy and the most common prior radiation was fractionated EBRT.

Histology of tumors was $85 \%$ adenocarcinoma (Table 2). Overall, roughly half of them occurred in the same hemithorax as the patient's initial lung cancer. A fractionation schedule of $50 \mathrm{~Gy}$ in five fractions was the most commonly employed regimen (Table 3 ), and the most common prescription regardless of target location was $50 \mathrm{~Gy}$ in five fractions. Of the 74 tumors, none of those centrally located were treated in a single fraction, whereas $29 \%$ of the peripherally located tumors were treated in one fraction. The centrally located tumors had a median PTV of 28 versus $19 \mathrm{~mL}$ for peripherally located tumors, but the ranges of tumor size were highly overlapping and the difference was not significant $(p=0.52)$.

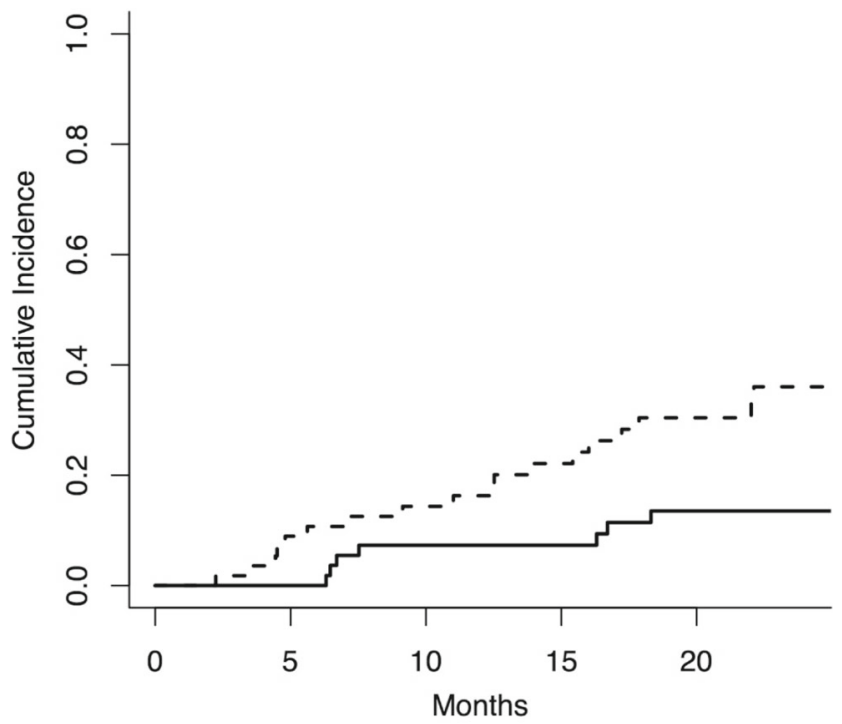

Number of Patients at Risk

$56 \quad 51$

$42 \quad 34$

24

Fig. 1 Cumulative incidence of local failure (solid) with death as a competing risk (dotted) among patients retreated with SBRT after a prior therapy for lung cancer. Months are calculated since retreatment SBRT

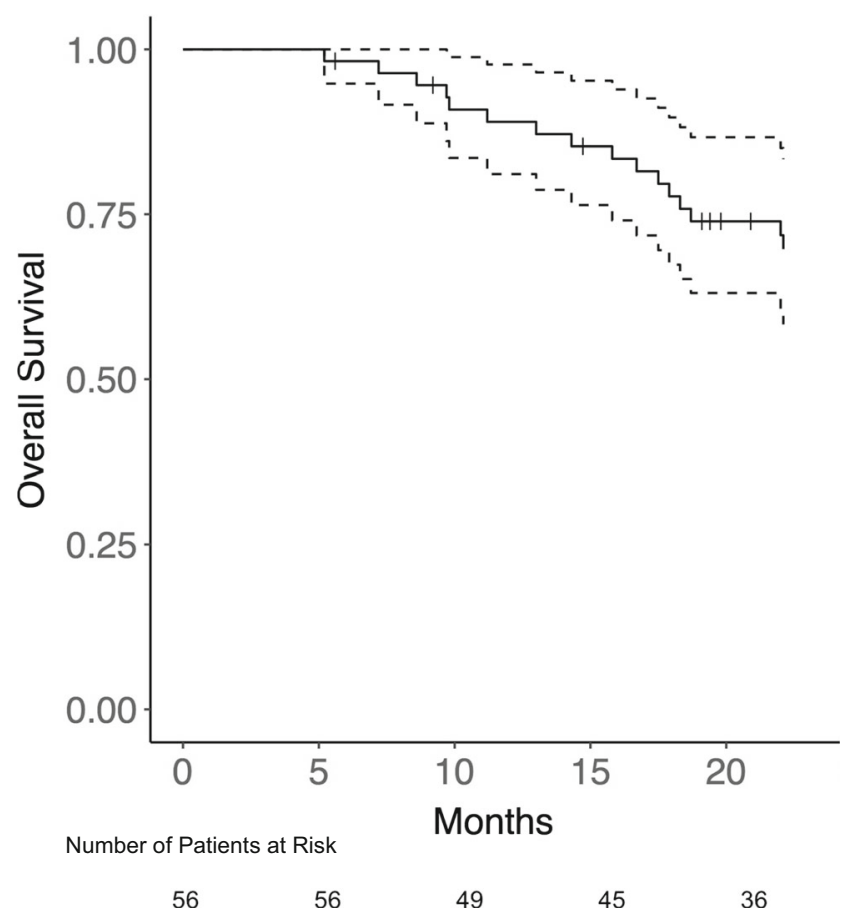

Fig. 2 Overall survival of retreatment SBRT patients with prior history of lung cancer. Ninety-five percent confidence intervals given in dotted lines. Months are calculated since SBRT retreatment

\section{Tumor control and overall survival}

There were eight local failures and 29 deaths at the time of analysis. At a median time of 27 months of follow-up, the 2year cumulative incidence rates of local failure were $14 \%$ overall (Fig. 1), 16\% for central tumors and 13\% for peripheral tumors (Gray's $p=0.95$ ). Two-year estimated overall survival rates were $70 \%$ overall (Fig. 2), $62 \%$ for central tumors and $72 \%$ for peripheral tumors (log-rank $p=0.48$ ). Neither a history of thoracic radiation nor a history of thoracic surgery had a negative impact on local tumor control or overall survival (Table 4). Treatment to multiple sites was associated with a higher risk of local failure with 1- and 2-year local failure incidences of 23 versus $2 \%$ and 38 versus $5 \%$ $(p=0.002)$, respectively, but was not associated with a higher risk of death $(p=0.72)$. The reasons for this difference in local control were likely twofold: (1) patients with multiple treated sites were inherently at greater risk of local failure to one of any treated sites and (2) biologically effective doses (BEDs) were often numerically lower in patients who were treated to multiple (median $=100$, range 36-151 Gy10) versus single sites (median $=100$, range $60-180$ Gy10), but the difference was of only borderline significance $(p=0.11)$ (Table 3 ).

\section{Toxicity}

Among 12 patients with $\geq$ grade 2 toxicities, grades 2,3 , and 4 toxicities occurred in eight patients, three patients, and one 
Table 4 Cumulative incidence of local failure in the presence of competing risk, death, and overall survival rates after retreatment SBRT, in 56 patients with history of non-small cell lung cancer retreated with stereotactic body radiation therapy

\begin{tabular}{|c|c|c|c|c|c|}
\hline & & Local failure (\%) & $p$ value & Overall survival (\%) & $p$ value \\
\hline \multirow[t]{2}{*}{ All patients } & 1 year & 7 & & 89 & \\
\hline & 2 year & 14 & & 70 & \\
\hline \multirow[t]{2}{*}{ Central } & 1 year & 7 & 0.95 & 86 & 0.48 \\
\hline & 2 year & 16 & & 62 & \\
\hline \multirow[t]{2}{*}{ Peripheral } & 1 year & 7 & & 90 & \\
\hline & 2 year & 13 & & 72 & \\
\hline \multirow[t]{2}{*}{ Prior thoracic radiation } & 1 year & 7 & 0.91 & 90 & 0.30 \\
\hline & 2 year & 15 & & 73 & \\
\hline \multirow[t]{2}{*}{ No prior thoracic radiation } & 1 year & 7 & & 89 & \\
\hline & 2 year & 12 & & 66 & \\
\hline \multirow[t]{2}{*}{ Prior surgery } & 1 year & 5 & 1.00 & 90 & 0.51 \\
\hline & 2 year & 14 & & 71 & \\
\hline \multirow[t]{2}{*}{ No prior surgery } & 1 year & 13 & & 88 & \\
\hline & 2 year & 13 & & 68 & \\
\hline \multirow[t]{2}{*}{ Single lesion } & 1 year & 2 & 0.002 & 90 & 0.72 \\
\hline & 2 year & 5 & & 70 & \\
\hline \multirow[t]{2}{*}{ Multiple lesions } & 1 year & 23 & & 85 & \\
\hline & 2 year & 38 & & 69 & \\
\hline
\end{tabular}

patient, respectively. The cumulative incidence rates of $\geq$ grade 2 toxicity at 1,6 , and 12 months were 5,14 , and $14 \%$, respectively (Fig. 3). Individual descriptions of these toxicities are provided in Table 5 .

No patients with $\mathrm{a}<20$ pack-year smoking history experienced an adverse reaction but patients with $\mathrm{a} \geq 50$ pack-year smoking history had increased rates of toxicity compared to those with less $(p=0.01)$ (Table 6$)$. Among patients with $\geq 50$ pack-year smoking history, the 1- and 6-month rates of $\geq$

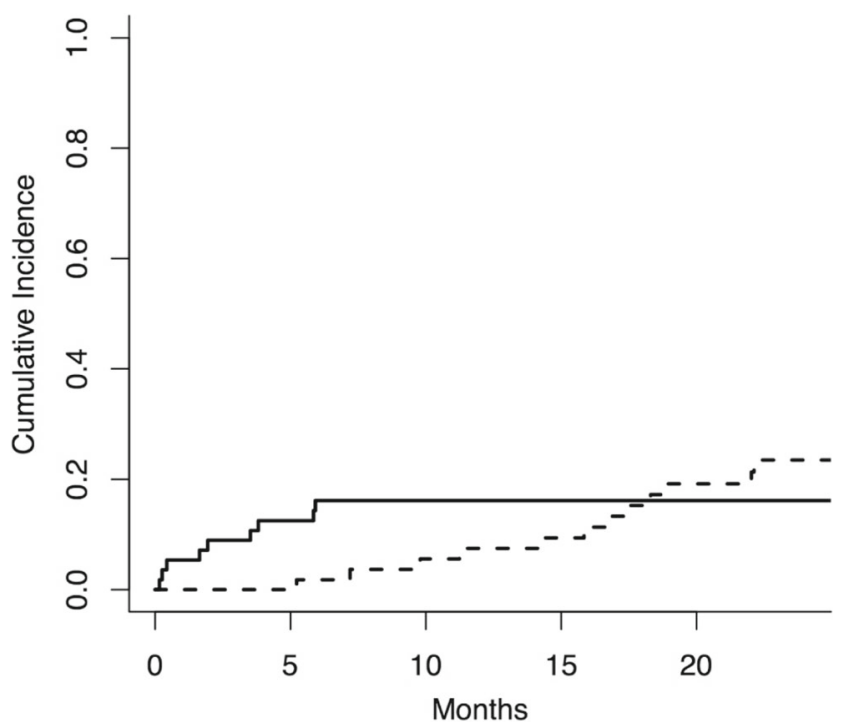

Number of Patients at Risk

$\begin{array}{lllll}56 & 49 & 41 & 38 & 31\end{array}$

Fig. 3 Cumulative incidence of grade $\geq 2$ toxicity (solid) with death as a competing risk (dotted), among patients retreated with SBRT after a prior therapy for lung cancer. Months are calculated since SBRT retreatment grade 2 toxicity were 8 and $30 \%$, respectively. Active smoking was not associated with increased toxicity $(p=0.96)$.

Advanced age, previous thoracic treatments, and treatment to multiple sites were not found to influence the incidence of toxicity (Table 6). However, centrally located tumors were associated with a higher rate of toxicity compared to peripheral tumors $(p=0.04)$. Among patients with centrally located tumors, the 1 - and 6-month rates of $\geq$ grade 2 toxicity were 7 and $36 \%$, respectively, versus 2 and $7 \%$ for peripheral tumors.

Larger planning target volumes (PTV) were also associated with higher rates of toxicity (Table 6). The 1- and 6-month rates of $\geq$ grade 2 toxicity for PTVs $30 \mathrm{~mL}$ or greater were 11 and $22 \%$, versus 0 and $11 \%$ for smaller PTVs $(p=0.047)$. There was no difference in the distribution of PTVs $(p=0.52)$ in central versus peripheral patients.

In a bivariate model including $\geq 50$ pack-year smoking history and PTV size $\geq 30 \mathrm{~mL}$, neither was significant (Table 7). However, in a multivariable model that included central tumor location versus $\geq 50$ pack-year smoking history and PTV size $\geq 30 \mathrm{~mL}$, central tumor location remained significant for influencing $\geq$ grade 2 toxicity $(p=0.0437)$.

\section{Discussion}

In our thoracic SBRT population, the majority of complications have occurred in patients with a prior history of lung cancer treatment. We sought to quantify the rate of toxicity specifically in this retreatment population and found that overall, $7 \%$ experienced $\geq$ grade 3 toxicity to SBRT and $14 \%$ experienced $\geq$ grade 2 toxicity. Patient age, active smoking, and the type of treatment delivered at first lung cancer diagnosis did not influence the 


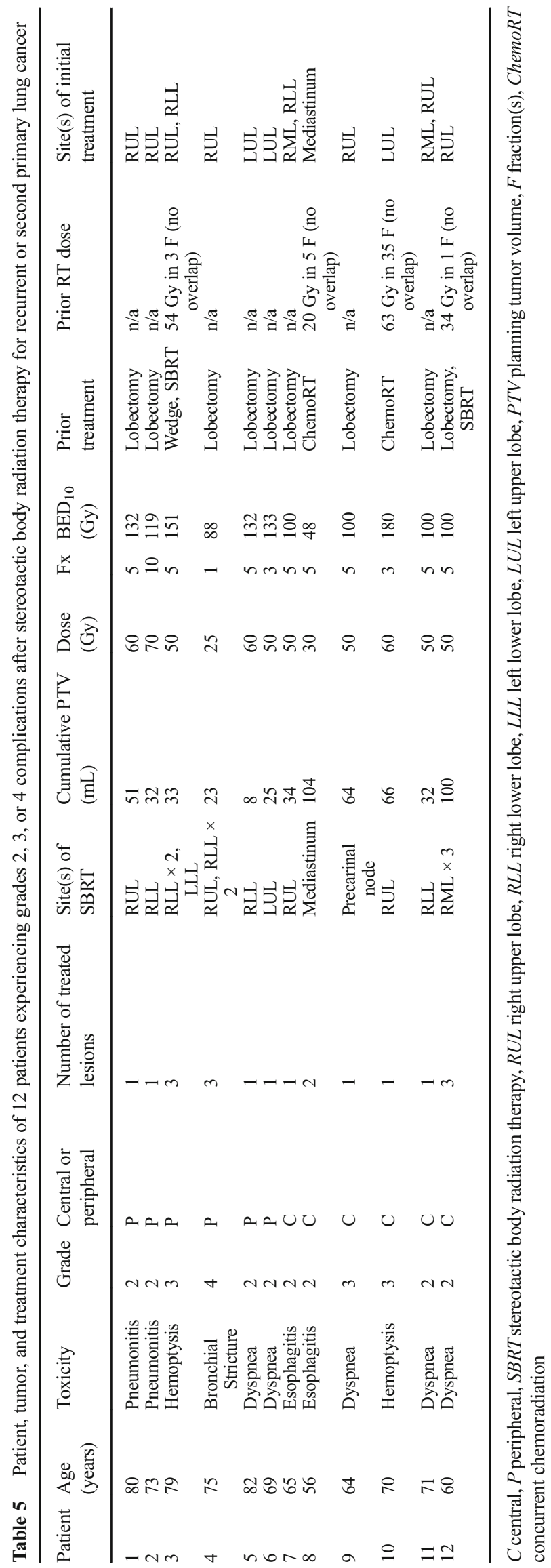

Table 6 Cumulative incidence of $\geq$ grade 2 toxicity among 56 patients with history of non-small cell lung cancer receiving SBRT retreatment, on univariate analysis by log-rank test

\begin{tabular}{|c|c|c|c|c|}
\hline & Months & $\begin{array}{l}\geq \text { Grade } 2 \\
\text { toxicity }(\%)\end{array}$ & $\begin{array}{l}\geq \text { Grade } 2 \\
\text { toxicity }(\%)\end{array}$ & $\begin{array}{l}p \\
\text { value }\end{array}$ \\
\hline \multirow[t]{3}{*}{ All patients } & 1 & 5 & & \\
\hline & 6 & 14 & & \\
\hline & 12 & 14 & & \\
\hline \multirow[t]{4}{*}{ Age } & & $<70$ years & $\geq 70$ years & 0.76 \\
\hline & 1 & 4 & 3 & \\
\hline & 6 & 16 & 13 & \\
\hline & 12 & 16 & 13 & \\
\hline \multirow[t]{12}{*}{ Smoking history } & & $<20$ pack-years & $\geq 20$ pack-years & 0.07 \\
\hline & 1 & 0 & 7 & \\
\hline & 6 & 0 & 21 & \\
\hline & 12 & 0 & 21 & \\
\hline & & $<50$ pack-years & $\geq 50$ pack-years & 0.01 \\
\hline & 1 & 3 & 8 & \\
\hline & 6 & 6 & 30 & \\
\hline & 12 & 6 & 30 & \\
\hline & & Active smoker & Not active & 0.96 \\
\hline & 1 & 0 & 7 & \\
\hline & 6 & 9 & 18 & \\
\hline & 12 & 9 & 18 & \\
\hline \multirow[t]{4}{*}{ Prior surgery } & & No & Yes & 0.29 \\
\hline & 1 & 0 & 6 & \\
\hline & 6 & 6 & 20 & \\
\hline & 12 & 6 & 20 & \\
\hline \multirow[t]{4}{*}{ Prior radiation } & & No & Yes & 0.18 \\
\hline & 1 & 10 & 0 & \\
\hline & 6 & 28 & 4 & \\
\hline & 12 & 28 & 4 & \\
\hline \multirow{4}{*}{$\begin{array}{l}\text { Ipsilateral prior } \\
\text { treatment }\end{array}$} & & No & Yes & 0.10 \\
\hline & 1 & 0 & 10 & \\
\hline & 6 & 8 & 23 & \\
\hline & 12 & 8 & 23 & \\
\hline \multirow[t]{4}{*}{ Lesion location } & & Central & Peripheral & 0.04 \\
\hline & 1 & 7 & 2 & \\
\hline & 6 & 36 & 7 & \\
\hline & 12 & 36 & 7 & \\
\hline \multirow[t]{4}{*}{ Lesion number } & & Single & Multiple & 0.48 \\
\hline & 1 & 7 & 0 & \\
\hline & 6 & 16 & 15 & \\
\hline & 12 & 16 & 15 & \\
\hline \multirow[t]{4}{*}{ PTV volume } & & $<30 \mathrm{~mL}$ & $\geq 30 \mathrm{~mL}$ & 0.05 \\
\hline & 1 & 0 & 11 & \\
\hline & 6 & 11 & 22 & \\
\hline & 12 & 11 & 22 & \\
\hline \multirow[t]{4}{*}{ Fraction number } & & $<5$ & $\geq 5$ & 0.47 \\
\hline & 1 & 0 & 9 & \\
\hline & 6 & 9 & 21 & \\
\hline & 12 & 9 & 21 & \\
\hline
\end{tabular}

PTV planning target volume

development of toxicity. Whether SBRT was delivered to single or multiple sites was also not associated with increased toxicity, although it should be noted that a lower BED was used in 
Table 7 Bivariate associations and multivariable model of competing risk regression of covariates associated with $\geq$ grade 2 toxicity, in 56 patients receiving lung cancer retreatment

\begin{tabular}{llll}
\hline Covariate & Hazard ratio & $95 \%$ confidence interval & $p$ value \\
\hline Bivariate associations & & & \\
$\quad$ Central lesion & 3.37 & $1.13-10.00$ & 0.03 \\
Smoking history $\geq 50$ pack-years & 4.61 & $1.26-16.90$ & 0.02 \\
Smoking history $\geq 50$ pack-years & 3.71 & $0.91-15.00$ & 0.07 \\
PTV volume $\geq 30 \mathrm{~mL}$ & 2.63 & $0.67-10.30$ & 0.17 \\
Central lesion & 3.34 & $1.14-9.83$ & 0.03 \\
PTV volume $\geq 30 \mathrm{~mL}$ & 2.99 & $0.81-11.02$ & 0.10 \\
Multivariable model & & & \\
Central lesion & & & 0.04 \\
Smoking history $\geq 50$ pack-years & 3.21 & $1.07-9.60$ & 0.08 \\
PTV volume $\geq 30 \mathrm{~mL}$ & 3.58 & $0.87-14.69$ & 0.25 \\
\hline
\end{tabular}

PTV planning target volume patients treated to multiple sites. However, rates of $\geq$ grade 2 toxicity were significantly higher on univariable analysis in patients with centrally located tumors, $\geq 50$ pack-year smoking history, or PTV $\geq 30 \mathrm{~mL}$. In all bivariate models and in limited multivariable analysis within the confines of this dataset, central tumor location remained significant.

There have been few prior reports explicitly evaluating the impact of tumor location on the safety and efficacy of SBRT in previously treated NSCLC patients. What has been extensively reported in the literature is the safety and durable tumor control associated with treating centrally located tumors, whether primary NSCLC or pulmonary metastases, with SBRT. For example, in 2013, Senthi et al. reviewed publications between 2010 and 2012 that included a total of 418 patients treated with SBRT for centrally located tumors and found that grade 3 or greater toxicity was only seen in $8.6 \%$ of patients. Even in patients with large primary $(\geq 5 \mathrm{~cm})$ NSCLC, a multi-institutional series found no differences in grade 2 or greater toxicities based on central versus peripheral tumor location [13]. In our dataset specifically focused on patients who have either had a prior lung surgery and/or course of thoracic radiation therapy, we found that the rates of toxicity were low among patients with peripheral lesions, but a central location of the new treatment predisposed for a much higher risk, with a 6-month $\geq$ grade 2 toxicity rate of $36 \%$. This was despite the common use of more conservative fractionation schedules for central tumors.

Our overall results are concordant with growing evidence that thoracic reirradiation to peripheral sites is reasonably safe using SBRT but caution is needed for central tumors (Table 8). In one of the earlier reports of thoracic reirradiation with SBRT, Peulen et al. found that SBRT was feasible but larger treatment volumes and centrally located tumors were risk factors for grades 3-5 toxicities [14]. Meijneke et al. reported a series of 20 patients who underwent thoracic reirradiation with SBRT as part of the first or second treatment, with no grades 3-5 toxicities observed
[15]. Binkley et al. reported a series of 38 patients reirradiated at a median time of 16 months after initial thoracic radiation. There were only seven cases of any grade 3 toxicity and no cases of $\geq$ grade 4 toxicity [16]. On the other hand, reirradiation to larger areas using conventional external beam therapy, whether photonor proton-based, resulted in higher risk, with rates of high-grade toxicity of 33-42\% [17] [18]. In our cohort, an SBRT planning tumor volume $>30 \mathrm{~mL}$ influenced development of $\geq$ grade 2 toxicity on univariable analysis but was not as important of a risk factor as central tumor location in multivariate analysis.

Our report is subject to the usual limitations of a retrospective cohort study, including incomplete or inaccurate toxicity grading and the potential for nonrandom missing data and follow-up. Another limitation is a lack of consistent functional lung assessment such that the degree of baseline impairment from prior surgery and/or radiation could not reliably be assessed at the time of patients' SBRT retreatment. The patients in this series were all treated in an experienced SBRT demonstration center, by clinicians who anticipated toxicity when designing the treatment plans, and the rates may not reflect what would have been seen in a population treated without regard to size and location.

We chose to include patients treated to multiple sites in this review, to provide a real-world analysis of this frequently encountered and difficult clinical situation, among a population whose treatment options are often very limited. In our study cohort, patients with central tumors were more likely to have treatment to multiple sites, and they also had larger PTV sizes, but their increased risks of toxicity were likely mitigated by the more conservative schedules used in these patients. Despite what might have been "gentler" schedules overall, centrality emerged as a major risk factor for severe toxicity.

It is possible that patients with centrally located tumors were more likely to have recurrent tumors as opposed to truly new second primary tumors, but we did not see greater rates of local failure or worsened survival associated with the factor of 
centrality. For practical reasons, we included all patients for whom there was a consensus by our thoracic group that they could have new second primary tumors, as the distinction between second primary and recurrent tumors is not often clinically evident. The 2-year overall survival rates of our patients, comparable to those of the patients in RTOG 0813 who were treated at initial presentation, indicate that SBRT retreatment can be a very effective therapy in the complex life cycle of these heavily treated patients.

Our study describes a common clinical scenario, provides useful benchmarks for severe toxicity and efficacy in this vulnerable population, and establishes central location, heavy smoking history, and large PTV size as major factors predisposing for risk. As the median survival of lung cancer patients of all stages improves, additional episodes of thoracic retreatment are becoming an increasingly common phenomenon. Our report provides practical data on the expected risks and benefits of retreatment SBRT for patients who do not have other options for management of a recurrent or second primary NSCLC.

\section{Conclusions}

SBRT for thoracic retreatment of NSCLC appears to result in acceptable rates of severe toxicity overall, but rates of $\geq$ grade 2 toxicities are increased in patients who have centrally located tumors, with heavy smoking history and large PTV size also contributing to risk.

\section{Compliance with ethical standards}

Funding No funding was used or received in the production of this work.

Conflict of interest Dr. Descovich receives research support from Accuray, Inc. Dr. Yom receives research support from Genentech, Bristol Myers-Squibb, and Merck. All other authors have no relevant conflicts of interest to disclose.

Ethical approval All procedures performed in studies involving human participants were in accordance with the ethical standards of the UCSF Institutional Review Board and with the 1964 Helsinki declaration and its later amendments or comparable ethical standards.

Informed consent For this type of study, formal consent was not required. Waiver of consent for medical chart review was approved by the Institutional Review Board (study number 10-05156).

Open Access This article is distributed under the terms of the Creative Commons Attribution 4.0 International License (http:// creativecommons.org/licenses/by/4.0/), which permits use, duplication, adaptation, distribution and reproduction in any medium or format, as long as you give appropriate credit to the original author(s) and the source, provide a link to the Creative Commons license, and indicate if changes were made. 


\section{References}

1. Boyle JM, Tandberg DJ, Chino JP et al (2015) Smoking history predicts for increased risk of second primary lung cancer: a comprehensive analysis. Cancer 121:598-604. https://doi.org/10.1002/ cncr.29095

2. Okamoto Y, Murakami M, Yoden E et al (2002) Reirradiation for locally recurrent lung cancer previously treated with radiation therapy. Int J Radiat Oncol Biol Phys 52:390-396

3. Hearn JWD, Videtic GMM, Djemil T, Stephans KL (2014) Salvage stereotactic body radiation therapy (SBRT) for local failure after primary lung SBRT. Int J Radiat Oncol Biol Phys 90:402-406. https://doi.org/10.1016/j.jirobp.2014.05.048

4. Trakul N, Harris JP, Le Q-T et al (2012) Stereotactic ablative radiotherapy for reirradiation of locally recurrent lung tumors. J Thorac Oncol 7:1462-1465. https://doi.org/10.1097/JTO. 0b013e31825f22ce

5. Chang JY, Senan S, Paul MA et al (2015) Stereotactic ablative radiotherapy versus lobectomy for operable stage I non-small-cell lung cancer: a pooled analysis of two randomised trials. Lancet Oncol 16:630-637. https://doi.org/10.1016/S1470-2045(15) 70168-3

6. Timmerman R, McGarry R, Yiannoutsos C et al (2006) Excessive toxicity when treating central tumors in a phase II study of stereotactic body radiation therapy for medically inoperable early-stage lung cancer. J Clin Oncol 24:4833-4839. https://doi.org/10.1200/ JCO.2006.07.5937

7. Senthi S, Haasbeek CJA, Slotman BJ, Senan S (2013) Outcomes of stereotactic ablative radiotherapy for central lung tumours: a systematic review. Radiother Oncol 106:276-282. https://doi.org/10. 1016/j.radonc.2013.01.004

8. Bezjak A, Paulus R, Gaspar LE et al (2016) Primary study endpoint analysis for NRG oncology/RTOG 0813 trial of stereotactic body radiation therapy (SBRT) for centrally located non-small cell lung cancer (NSCLC). Int J Radiat Oncol Biol Phys 94:5-6. https://doi. org/10.1016/j.ijrobp.2015.10.040
9. Benedict SH, Yenice KM, Followill D et al (2010) Stereotactic body radiation therapy: the report of AAPM task group 101. Med Phys 37:4078-4101. https://doi.org/10.1118/ 1.3438081

10. Fine JP, Gray RJ (1999) A proportional hazards model for the subdistribution of a competing risk. J Am Stat Assoc 94:496. https://doi.org/10.2307/2670170

11. Kaplan EL, Meier P (1958) Nonparametric estimation from incomplete observations. J Am Stat Assoc 53:457. https://doi.org/10. 2307/2281868

12. Cox DR (1972) Regression models and life-tables. J R Stat Soc Ser B Methodol 34:187-220

13. Verma V, Shostrom VK, Kumar SS et al (2017) Multi-institutional experience of stereotactic body radiotherapy for large ( $\geq 5$ centimeters) non-small cell lung tumors. Cancer 123:688-696. https://doi. org/10.1002/cncr.30375

14. Peulen H, Karlsson K, Lindberg K et al (2011) Toxicity after reirradiation of pulmonary tumours with stereotactic body radiotherapy. Radiother Oncol 101:260-266. https://doi.org/10.1016/j. radonc.2011.09.012

15. Meijneke TR, Petit SF, Wentzler D et al (2013) Reirradiation and stereotactic radiotherapy for tumors in the lung: dose summation and toxicity. Radiother Oncol 107:423-427. https://doi.org/10. 1016/j.radonc.2013.03.015

16. Binkley MS, Hiniker SM, Chaudhuri A et al (2016) Dosimetric factors and toxicity in highly conformal thoracic reirradiation. Int J Radiat Oncol Biol Phys 94:808-815. https://doi.org/10.1016/j. ijrobp.2015.12.007

17. Dickhoff C, Senan S, Dahele M (2017) Radical-intent treatment of lung cancer after prior thoracic radiotherapy. J Thorac Oncol 12: e26-e27. https://doi.org/10.1016/j.jtho.2016.12.009

18. Chao H-H, Berman AT, Simone CB et al (2017) Multiinstitutional prospective study of reirradiation with proton beam radiotherapy for locoregionally recurrent non-small cell lung cancer. J Thorac Oncol 12:281-292. https://doi.org/10. 1016/j.jtho.2016.10.018 